\title{
Estudo morfológico de Triatoma guazu Lent \& Wygodzinsky, 1979 (Hemiptera, Reduviidae, Triatominae)
}

\author{
Itamar Galindez-Girón, Rodolfo U Carcavallo*, José Jurberg**/+, \\ Herman Lent***, Ortrud Monika Barth****
}

\begin{abstract}
Universidade de Los Andes, Núcleo Rafael Rangel, Laboratório de Biologia de Triatominae, Trujillo Venezuela *CIBION/COMICET, Serrano 669 (1414), Buenos Aires, Argentina **Laboratório Nacional e Internacional de Referência em Taxonomia de Triatomíneos, Departamento de Entomologia, Instituto Oswaldo Cruz, Av. Brasil 4365, 21045-900 Rio de Janeiro, RJ, Brasil ***Centro de Ciências Biológicas, Universidade Santa Úrsula,

Rio de Janeiro, Brasil ****Departamento de Virologia, Instituto Oswaldo Cruz, Rio de Janeiro, RJ, Brasil
\end{abstract}

Morphologic Study of Triatoma guazu Lent \& Wygodzinsky, 1979 (Hemiptera, Reduviidae, Triatominae) - A female of Triatoma guazu Lent \& Wygodzinsky, 1979, a rare species from which the allotype was recently described, was studied by scanning electronic microscopy and videomicroscope. Some structures of the head and the thorax are shown. Some of them could have taxonomic importance, as the oculo-ocellar region, the buccula, the anterolateral angle of the collar, the scutellum, wings and the stridulatory sulcus, which has an unusual lateral high rim.

Key words: Triatoma guazu - anatomia microscópica - taxonomia

Em 1979 Lent e Wygodzinsky descreveram Triatoma guazu baseados no único exemplar fêmea capturado em 1934, em Villa Rica, na região oriental do Paraguai por F Schade (Lent \& Wygodzinsky 1979). Desde então, nenhum outro exemplar foi capturado, até que em 1994 o Laboratório Nacional e Internacional de Referência em Taxonomia de Triatomíneos (LNIRTT), Instituto Oswaldo Cruz, recebeu da Fundação Nacional de Saúde alguns exemplares mortos coletados em uma casa em Barra da Garça, Mato Grosso, Brasil. Posteriormente outros exemplares vivos, coletados na mesma área, foram incorporados ao Insetário de Triatomíneos do LNIRTT, onde estão sendo criados. O alótipo macho foi descrito por Lent et al. (1996) com ênfase nas estruturas fálicas. Agora complementamos a descrição com estudos morfológicos através de microscopia eletrônica de varredura e vídeo microscopia com o intuito de ampliar o conceito morfológico da espécie e as possibilidades de integrá-las em uma análise filogenética baseada na correlação com parâmetros fálicos (Jurberg 1996).

Realizado com auxílio do CNPq, Convênio 027/93 BIRD/FNS/FIOCRUZ, European Comission IC18-CT 960042.

${ }^{+}$Autor correspondente. Fax: +55-21-290.9339

Recebido em 23 de janeiro de 1997

Aceito em 12 de maio de 1997

\section{MATERIAIS E MÉTODOS}

Um exemplar fêmea da série coletada em 1994 em Barra da Garça, Mato Grosso, foi analisado macroscopicamente, no Instituto de NeurobilogiaCONICET de Buenos Aires, através de um vídeo microscópio Olympus OUM 1000 com um videoprinter SONY UP 3000. Os detalhes das estruturas foram analisados em um microscópio eletrônico de varredura JEOL JSM-35 CF e fotografados com aumentos de $20 \mathrm{X}$ a $360 \mathrm{X}$. O material foi previamente limpo com acetona; evitou-se usar o ultra-som e a metalização a fim de proteger o exemplar devido à sua raridade. Foram estudadas: cabeça vista dorsal e ventral, clípeo e anticlípeo, terceiro segmento do rostro, região óculo-ocelar, sinlipsis, bucula, genas, vista dorsal e ventral, tubérculos anteníferos, segundo artículo antenal, sulco estridulatório, escutelo, um par de coxas e o conexivo em vista ventral. Posteriormente foram feitos estudos complementares das asas no Departamento de Virologia da Fundação Oswaldo Cruz com microscópio eletrônico de varredura Zeiss-DSM-940.

\section{RESULTADOS}

Cabeça com clípeo alongado e dilatado na região posterior, tubérculos anteníferos com projeções sobressalentes apicais, região ante-ocular maior que pós-ocular, sinlipsis muito convexa e enrugada na parte mediana. Olhos proeminentes e os ocelos situados em elevações com aspecto divergente (Figs. 1 e 3). 


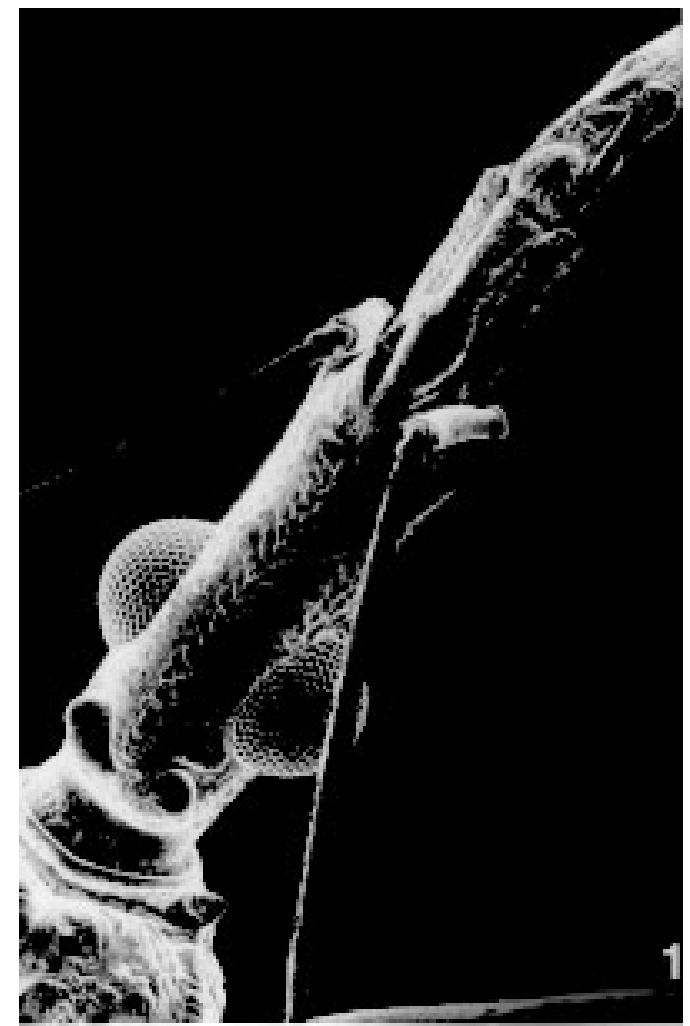

Fig. 1: clípeo, genas e labro, MEV 100X.

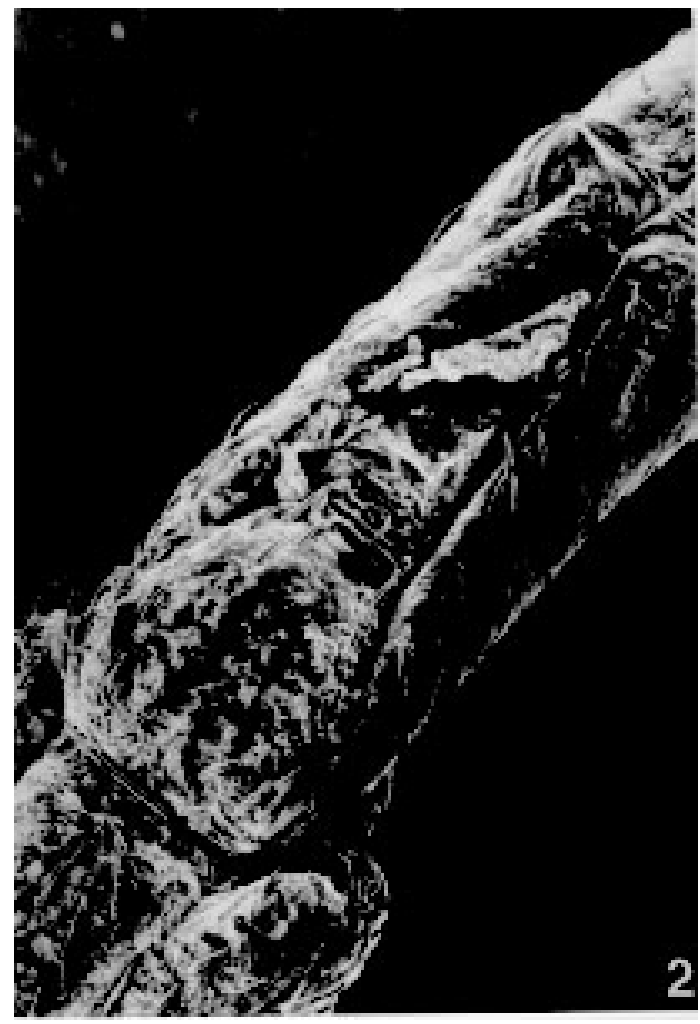

Fig. 2: cabeça vista dorsal de Triatoma guazu, MEV 24X.

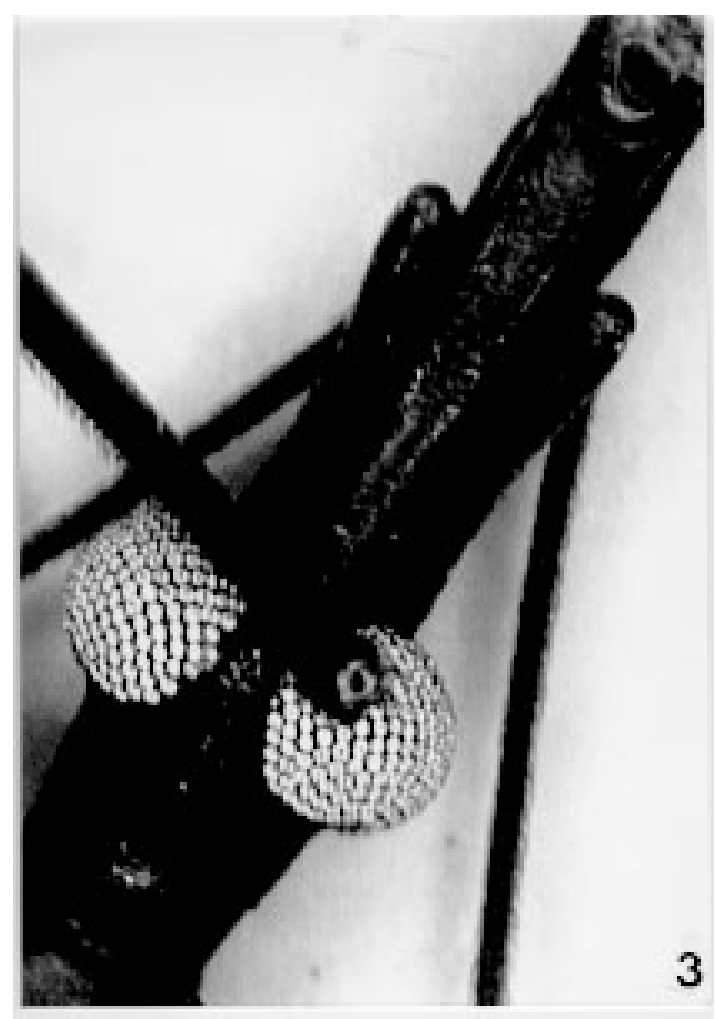

Fig. 3: cabeça em vista ventral, videomicrosc. 50X.

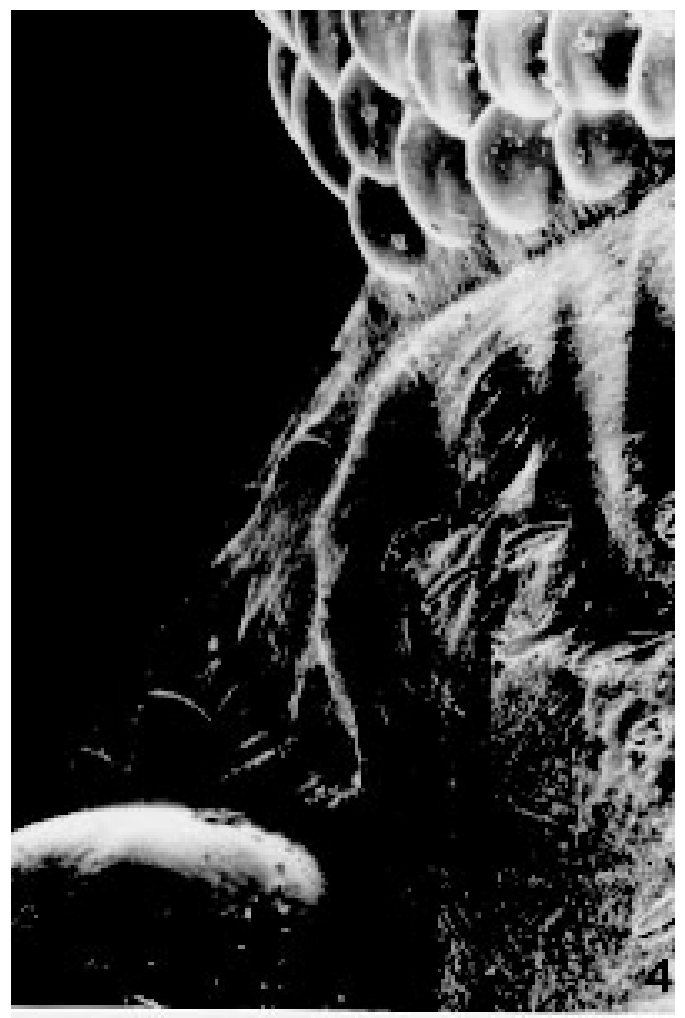

Fig. 4: região óculo-ocelar, MEV 300X. 
Anteclípeo de formato hexagonal de bordos e ângulos abaulados; a articulação com o clípeo é maior que a articulaçãpo com o labro, este último se aloja numa depressão de bordos glabros com poucos pêlos grossos e decumbentes, genas arredondadas (Fig. 2).

Região óculo-ocelar com tegumento liso, levemente pregueada com raros pêlos grossos na área central, áreas laterais glabras constituindo um sulco que une o olho ao ocelo, rodeando-os (Fig. 4).

Bucula com uma concavidade anterior acentuada, abrangendo várias estruturas da articulação do primeiro segmento do rostro, se continua numa área levemente deprimida, estreitando-se ao longo do comprimento até a região inter-ocular (Figs. 3 e 5).

Ângulo ântero-lateral saliente, cônico, com numerosos pêlos grossos com exceção da área ântero-superior que possui uma forma arredondada com aspecto de uma fosseta glabra; uma carena une o limite posterior do ângulo até o lóbulo anterior do pronoto (Fig. 6).

Escutelo cordiforme com a base e a área central bastante enrugada e o processo curto com ápice arredondado (Fig. 7).

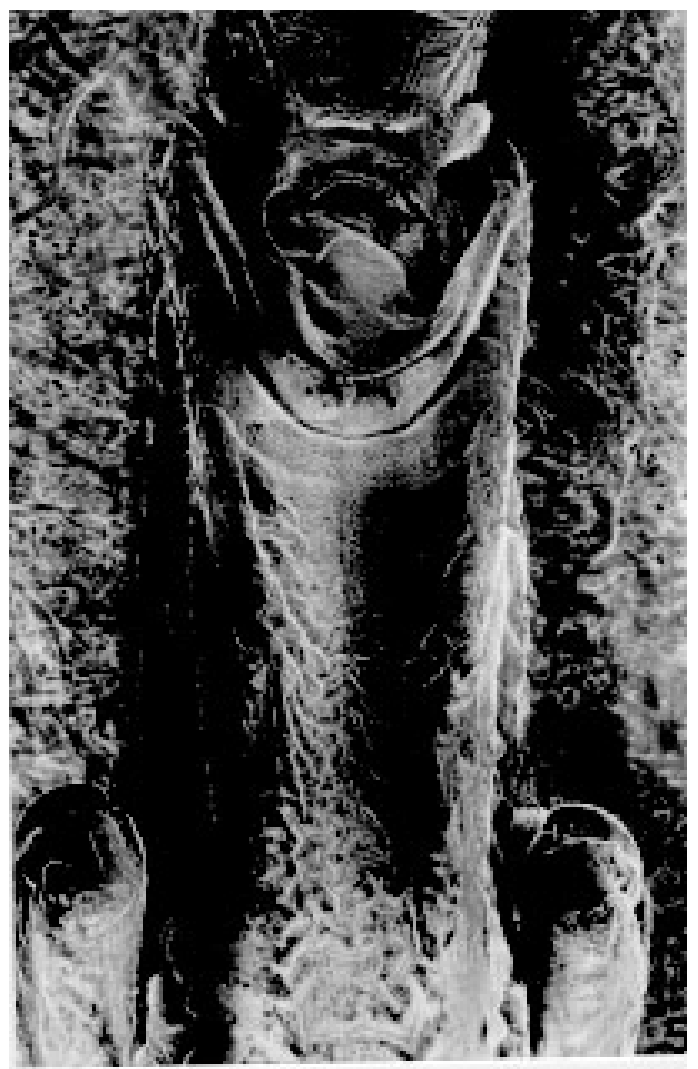

Fig. 5: região anterior da cabeça, com rostro estendido, vista ventral, MEV 86X.

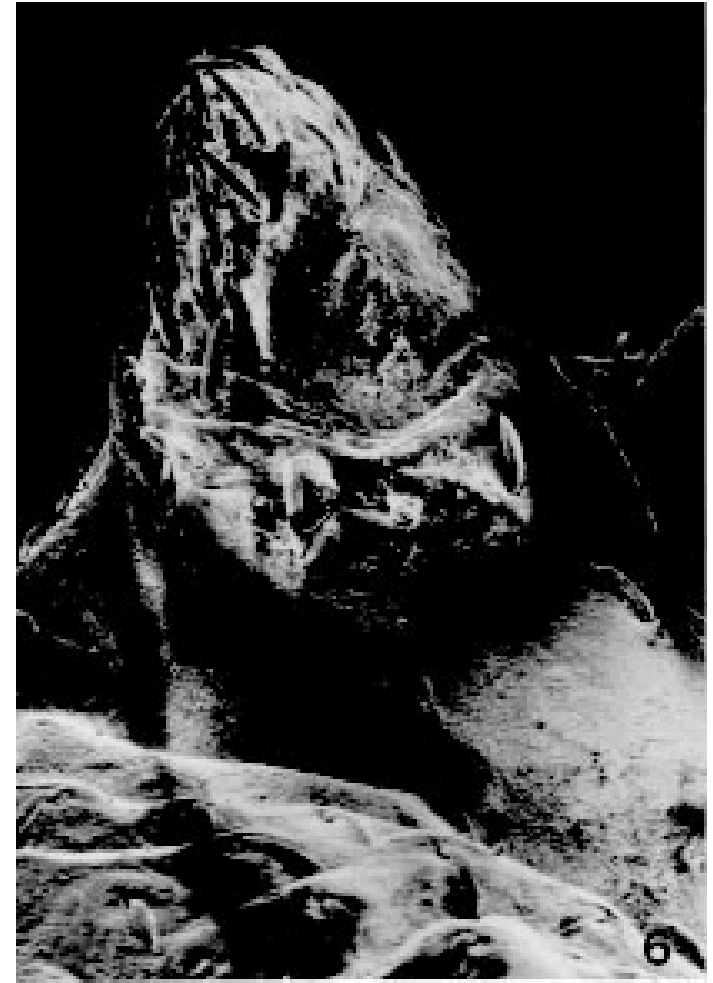

Fig. 6: ângulo antero-lateral do colar, MEV 240X.

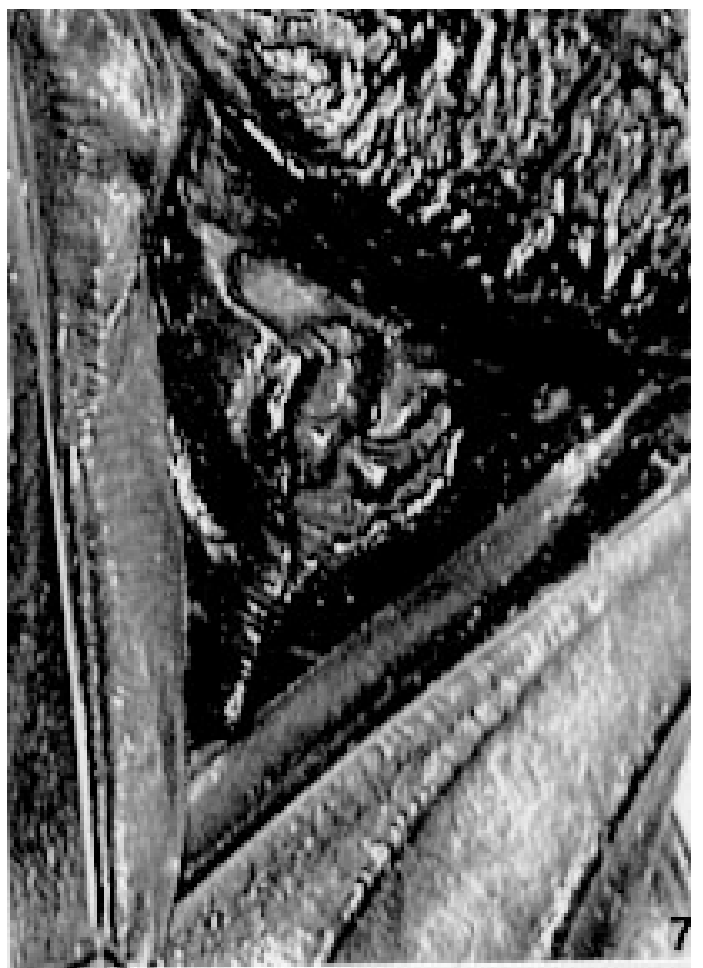

Fig. 7: escutelo, videomicrosc. 50X. 
Sulco estridulatório constituído por ondulações pouco salientes, bordos laterais muito elevados com numerosos pêlos grossos (Fig. 8).

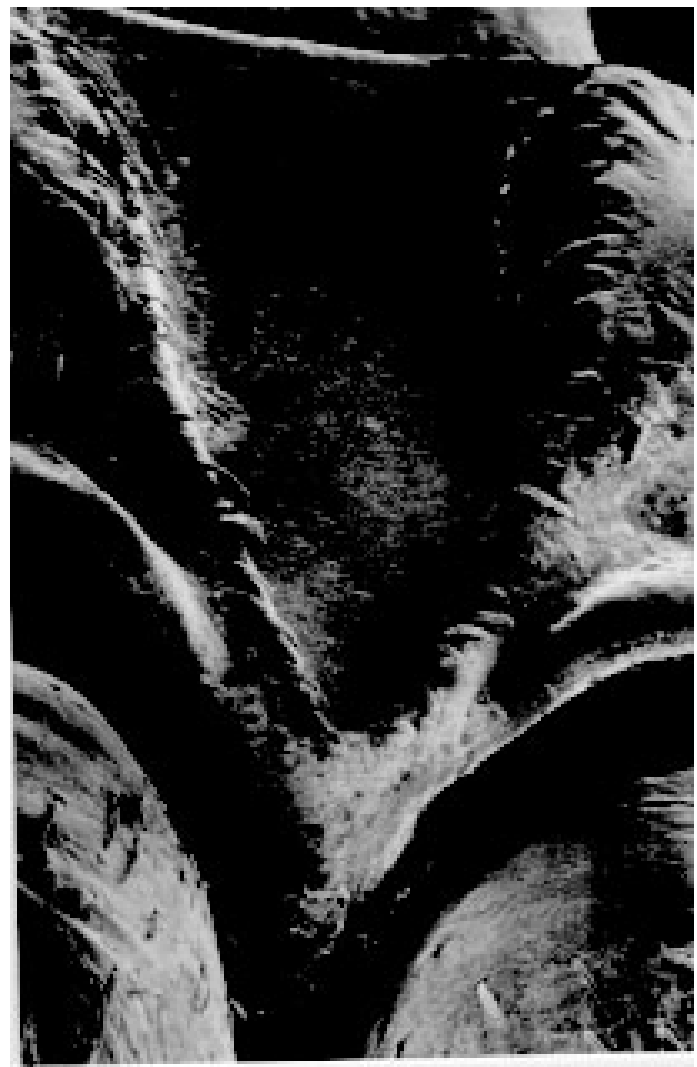

Fig. 8: sulco estridulatório, MEV 130X.

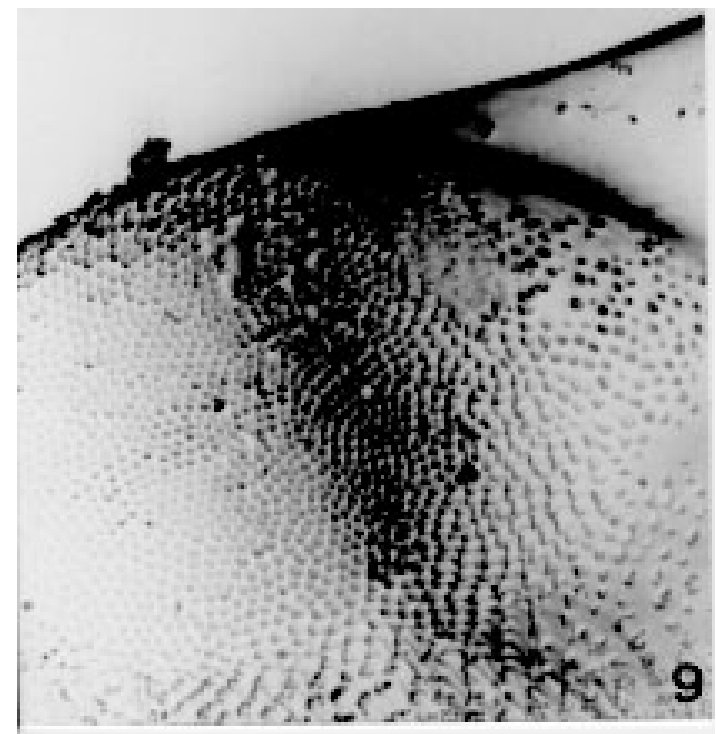

Fig. 9: asa - membrana, MEV 200X.
As asas evidenciaram na face externa um aspecto granuloso na membrana (Fig. 9), pêlos grossos decumbentes sobre o cório e o clavo (Fig. 10), e na face interna um coaptor cujo ctenídio com 33 cerdas se opõe a uma estrutura elevada recoberta de projeções retangulares de bordo superior liso. Entre as duas estruturas existe um vão aprofundado onde se encaixa a veia subcostal da membrana (Fig. 11).

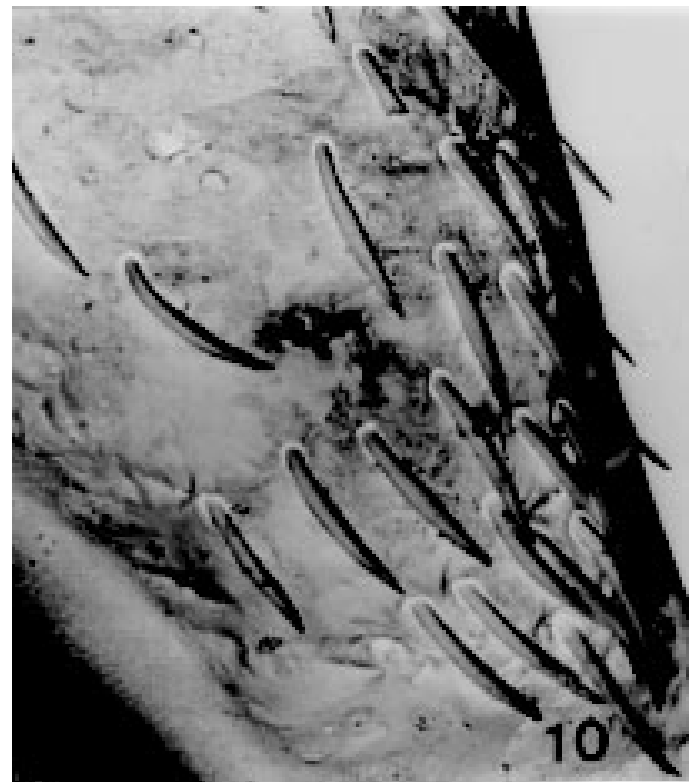

Fig. 10: asa - face externa do cório, MEV 200X.

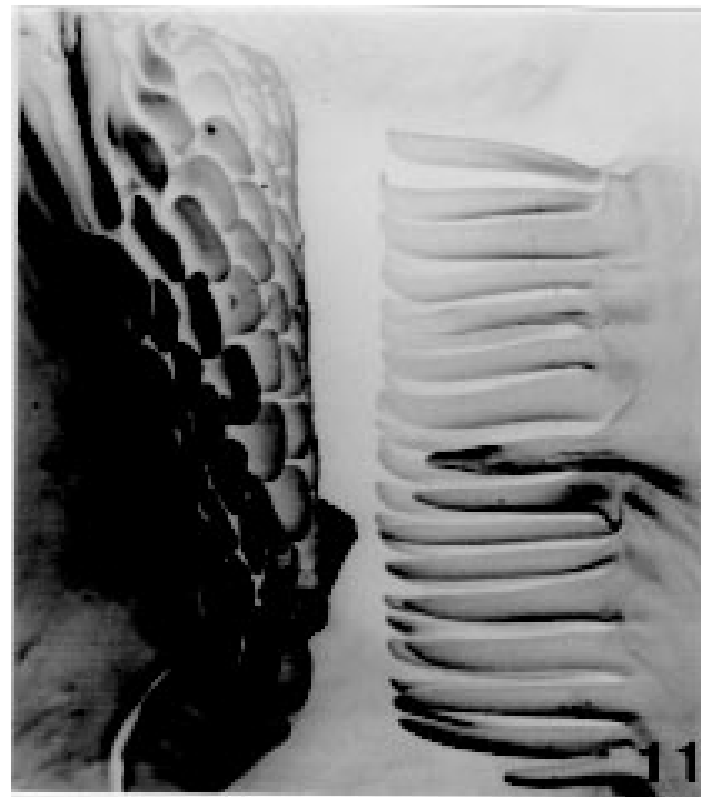

Fig. 11: asa - face interna do hemiélitro. Coaptor com típicos ctenídeos, MEV 500X 


\section{DISCUSSÃO E CONCLUSÃO}

As estruturas cuticulares evidenciadas ampliam o conhecimento desta espécie e permitem abordagens intra e inter-específicas. Estudos prévios sobre a região óculo-ocelar nos gêneros Psammolestes, Rhodnius e Panstrongylus sobre a importância das ornamentações estruturais nas diversas áreas do tegumento e do escutelo (Carcavallo et al. 1994 a,b,c, Carcavallo \& Galindez-Girón 1995), confirmam o valor que estas abordagens podem ter na caracterização específica, podendo ser incluída nas novas chaves dicotômicas, pois o tipo de pilosidade, distância entre o olho e ocelo, forma e direção do sulco retro-ocular, ornamentação na synlipsis, variação ornamental do tubérculo antenífero e do escutelo e o sulco estridulatório são detalhes de valor taxonômico de recente valorização.

\section{REFERÊNCIAS}

Carcavallo RU, Galindez-Girón I 1995. Systematic value of the scutellum in Triatominae (Hemiptera, Reduviidae). Entomol Vect 2: 23-29.
Carcavallo RU, Jurberg J, Martinez A, Galindez-Girón I 1994a. La región óculo-ocelar en el género Rhodnius Stal, 1859 (Hemiptera, Reduviidae, Triatominae). Entomol Vect 1: 21-32.

Carcavallo RU, Galindez I, Martinez A, Jurberg J 1994b. Cuticular structures of Triatominae. Their taxonomic significance. Entomol Vect 1: 79-91.

Carcavallo RU, Galindez-Girón I, Jurberg J, Martinez A 1994c. La región óculo-ocelar en el género Panstrongylus Berg, 1879 (Hemiptera, Reduviidae, Triatominae). Entomol Vect 1: 113-120.

Jurberg J 1996. Uma abordagem filogenética entre os Triatomíneos baseada nas estruturas fálicas, p. 116. Proc Intern Workshop Popul Genet Control Triatominae. IMDRE, México.

Lent H, Wygodzinsky P 1979. Revision of the Triatominae (Hemiptera, Reduviidae) and their significance as vectors of Chagas disease. Bull Am Mus Nat Hist 163: 125-520.

Lent H, Jurberg J, Galvão C 1996. Descrição do alótipo (macho) de Triatoma guazu Lent \& Wygodzinsky, 1979 proveniente do Estado do Mato Grosso, Brasil (Hemiptera, Reduviidae). Mem Inst Oswaldo Cruz 91: 313-315. 
544 Estudo morfológico de Triatoma guazu Lent \& Wygodzinsky, 1979 • I Galindez-Girón et al. 